\title{
Ceramide Biogenesis Is Required for Radiation-Induced Apoptosis in the Germ Line of C. elegans
}

\author{
Xinzhu Deng ${ }^{1}$, Xianglei Yin ${ }^{1}$, Richard Allan ${ }^{1}$, Diane D. Lu ${ }^{1}$, Carine W. Maurer ${ }^{2}$, Adriana \\ Haimovitz-Friedman ${ }^{3}$, Zvi Fuks ${ }^{3}$, Shai Shaham ${ }^{2}$, and Richard Kolesnick ${ }^{1,{ }^{*}}$ \\ 1 Laboratory of Signal Transduction, Memorial Sloan-Kettering Cancer Center (MSKCC), New York, NY \\ 10021, USA \\ 2Laboratory of Developmental Genetics, Rockefeller University, New York, NY, 10021, USA \\ 3Department of Radiation Oncology, Memorial Sloan-Kettering Cancer Center, New York, NY 10021, USA
}

\section{Abstract}

Ceramide engagement in apoptotic pathways has been a topic of controversy. To address this controversy, we tested loss-of-function ( $l f$ ) mutants of conserved genes of sphingolipid metabolism in Caenorhabditis elegans. Although somatic (developmental) apoptosis was unaffected, ionizing radiation-induced apoptosis of germ cells was obliterated upon inactivation of ceramide synthase and restored upon microinjection of long-chain natural ceramide. Radiation-induced increase in the concentration of ceramide localized to mitochondria and was required for BH3-domain protein EGL-1-mediated displacement of CED-4 (an APAF-1-like protein) from the CED-9 (a Bcl-2 family member)/CED-4 complex, an obligate step in activation of the CED-3 caspase. These studies define CEP-1 (the worm homolog of the tumor suppressor p53)-mediated accumulation of EGL-1 and ceramide synthase-mediated generation of ceramide through parallel pathways that integrate at mitochondrial membranes to regulate stress-induced apoptosis.

Although studies that use genetic deficiency in ceramide production support it as essential for apoptosis in diverse models (1), many have questioned whether ceramide functions as a bona fide transducer of apoptotic signals (2). One reason for skepticism is that, despite delineation of a number of ceramide-activated proteins, no single protein has been identified as mediator of ceramide-induced apoptosis. Recent studies have suggested an alternate mode of ceramide action, based on its capacity to self-associate and locally rearrange membrane bilayers into ceramide-rich macrodomains ( 1 to $5 \mu \mathrm{m}$ in diameter), which are sites of protein concentration and oligomerization (3). Ceramide may thus mediate apoptosis through its ability to reconfigure membranes, coordinating protein complexation at critical junctures of signaling cascades.

To establish the role of ceramide definitively, we used a model of radiation-induced apoptosis in Caenorhabditis elegans germ cells (4). Germline stem cells, located at the distal gonad tip, divide incessantly throughout adult life, with daughter cells arresting in meiotic prophase. Upon exiting prophase, germ cells become sensitive to radiation-induced apoptosis, detected morphologically just proximal to the bend of the gonadal arm (5). This apoptotic pathway is antagonized by the ABL-1 tyrosine kinase, requiring sequentially the cell cycle checkpoint genes rad-5, hus-1, and mrt-2; the C. elegans p53 homolog cep-1; and the genes making up the conserved apoptotic machinery, the caspase $c e d-3$, the apoptotic protease activating factor

*To whom correspondence should be addressed. E-mail: r-kolesnick@ski.mskcc.org. Supporting Online Material www.sciencemag.org/cgi/content/full/322/5898/110/DC1 
1-like protein ced-4, the Bcl-2 protein ced-9, and the BH3-domain protein egl-1. This pathway differs from apoptotic somatic cell death, which is not subject to upstream checkpoint regulation via the CEP-1 pathway $(5,6)$.

We identified conserved genes that regulate $C$. elegans sphingolipid intermediary metabolism and tested deletion alleles (Table 1 and table S1). Screening for mutants resistant to radiationinduced germ cell apoptosis revealed apoptosis suppression in only deletion mutants of hyl-1 and lagr-1, two of the three ceramide synthase (CS) genes (Fig. 1A). CS gene products regulate de novo ceramide biosynthesis, acylating sphinganine to form dihydroceramide that is subsequently converted to ceramide by a desaturase (7). CSs contain six to seven putative transmembrane domains and a Lag1p motif [which confers enzyme activity (8)], regions conserved in the C. elegans orthologs. The deleted CS sequences in hyl-1(ok976) and lagr-1 ( $g k 327)$ result in frameshifts that disrupt the Lag1p motifs (fig. S1A). We detected a $\sim 1.6-\mathrm{kb}$ hyl-1 transcript in wild-type (WT) worms and a smaller $\sim 1.35$-kb transcript in hyl-1(ok976), whereas we observed a $\sim 1.4$-kb lagr-1 transcript in WT worms and a $\sim 1.25$-kb transcript in lagr-1(gk327) (fig. S1B). In contrast, a deletion mutant of the third C. elegans CS $(9,10)$, hyl-2 (ok1766), lacking a 1626-base pair fragment of the hyl-2 gene locus that eliminates exons 2 to 5 corresponding to $74 \%$ of the coding sequence, displayed no defect in germ cell death (fig. S1C).

In N2 WTstrain young adults, apoptotic germ cells gradually increased in abundance with age from a baseline of $0.7 \pm 0.1$ to $1.8 \pm 0.2$ corpses per distal gonad arm over 48 hours. Exposure to a 120-gray (Gy) ionizing radiation dose increased germ cell apoptosis to $5.2 \pm 0.3$ cells 36 to 48 hours after treatment. In contrast, in hyl-1 (ok976) and lagr-1(gk327) animals, agedependent and radiation-induced germ cell apoptosis were nearly abolished (Fig. 1A). Similar effects were observed in the lagr-1(gk327); hyl-1(ok976) double mutant (Fig. 1B). The rate of germ cell corpse removal was unaffected in CS mutants, excluding the possibility that defective corpse engulfment elevated corpse numbers (table S2). In contrast, loss-of-function (lf) mutations of hyl-1 or lagr-1 did not affect developmental somatic cell death, nor did the lf hyl-2 (ok1766) mutation (table S3). These studies indicate a requirement for two C. elegans CS genes for radiation-induced germline apoptosis.

To confirm ceramide as critical for germline apoptosis, we injected $\mathrm{C}_{16}$-ceramide into gonads of young adult WT worms. $\mathrm{C}_{16}$-ceramide is the predominant ceramide species in apoptosis induction by diverse stresses in multiple organisms (11) and in low abundance in C. elegans $(12,13)$. $\mathrm{C}_{16}$-ceramide microinjection resulted in time- and dose-dependent increases in germ cell apoptosis (Fig. 1C), with a median effective dose of $\sim 0.05 \mu \mathrm{M}$ gonadal ceramide. Peak effect occurred at $\sim 0.1 \mu \mathrm{M}$ gonadal ceramide at 36 hours $(6.6 \pm 0.8$ versus $1.5 \pm 0.4$ cell corpses per distal gonad arm, $P<0.0001$ ), qualitatively and quantitatively mimicking the 120 -Gy effect in WT worms. In contrast, $\mathrm{C}_{16}$-dihydroceramide, which differs from $\mathrm{C}_{16}$-ceramide in a trans double bond at sphingoid base position four to five, was without effect $(0.71 \pm 0.28$ cell corpses per distal gonad arm at $\sim 1 \mu \mathrm{M}$ ), indicating specificity for ceramide in apoptosis induction. Furthermore, $\mathrm{C}_{16}$-ceramide microinjection into lagr-1(gk327);hyl-1(ok976) animals $(\sim 1 \mu \mathrm{M}$ gonadal ceramide) resulted in a 5.7-fold increase in germ cell apoptosis (from $0.60 \pm 0.17$ to $3.43 \pm 0.88, P<0.0001$ ) (Fig. 1D). Note that the baseline level of apoptosis in lagr-1 ( $g k 327)$; hyl-1( ok976) was less than one-half that in WT worms. Moreover, $\sim 0.005 \mu \mathrm{M}$ gonadal ceramide, a concentration without impact on germ cell apoptosis, completely restored radiation (120 Gy)-induced apoptosis, an effect inhibitable in a lf ced-3 background (Fig. 1E). $\mathrm{C}_{16^{-}}$ ceramide's ability to bypass the genetic defect and restore the radiation-response phenotype is strong evidence that hyl- 1 and lagr- 1 represent legitimate $C$. elegans CS genes. Animals with sphk-1(ok1097), a null allele of sphingosine kinase (SPHK), which prevents conversion of ceramide to its anti-apoptotic derivative sphingosine 1-phosphate (S1P) (14), displayed high baseline germ cell death and were hypersensitive to radiation-induced germ cell apoptosis (fig. 
S2, A and B), inhibitable (by $85 \pm 9 \%$ ) in a lagr-1( $g k 327) ; s p h k-1$ (ok1097) double mutant. Collectively, these studies identify ceramide as a critical effector of radiation-induced germ cell apoptosis, although they do not define its mode of engaging the apoptotic pathway.

Inactivation of the C. elegans ABL-1 ortholog in the $1 f$ mutant abl-1(ok171) (or by RNA interference) increases baseline and post-radiation germ cell apoptosis, modeling radiation hypersensitivity phenotypes (15). To order CS action relative to ABL-1, we generated hyl-1 (ok976);abl-1(ok171) and lagr-1 (gk327);abl-1(ok171) and a triple mutant lagr-1 (gk327);hyl-1 (ok976);abl-1(ok171). If hyl-1 or lagr-1 in an abl-1(ok171) genetic background prevented the time-dependent increase in physiologic germ cell apoptosis and completely blocked radiationinduced apoptosis (Fig. 2A, left). Similarly, lagr-1(gk327);hyl-1(ok976);abl-1(ok171) displayed inhibition of baseline and radiation-induced germ cell apoptosis (Fig. 2A, right). Thus, increased germ cell apoptosis in irradiated $a b l-1(o k 171)$ depends on the CS genes hyl-1 and lagr-1.

In C. elegans, DNA damage activates the p53 homolog CEP-1, which is required for transcriptional up-regulation of the BH3-only proteins, EGL-1 and CED-13, that in turn activate the core apoptotic machinery (CED-9, CED-4, and CED-3) $(6,16)$. Exposure of hyl-1 (ok976) and lagr-1 ( $g k 327$ ) to $120 \mathrm{~Gy}$ increased egl-1 transcripts four- to fivefold at 9 hours after irradiation (Fig. 2B, left), whereas ced-13 expression was enhanced five- to sixfold (Fig. 2B, right)-levels comparable to those detected in irradiated WTworms. Thus, the loss of CS did not affect CEP-1 activation upon irradiation, suggesting that ceramide and CEP-1 might function in parallel, coordinately conferring radiation-induced germ cell death.

We reasoned that in contrast to radiation-induced germ cell apoptosis, which apparently requires increased abundance of both $\mathrm{BH} 3$-only proteins and ceramide, $\mathrm{C}_{16}$-ceramide provided exogenously might act independent of p53-mediated egl- 1 expression by maximizing the effect of baseline EGL-1. In fact, microinjected $\mathrm{C}_{16}$-ceramide partially restored germ cell death in cep-1 $(g k 138)$ from $0.4 \pm 0.13$ to $2.5 \pm 0.32$ corpses per distal gonad arm (Fig. $2 \mathrm{C})(P<0.001)$. As $\mathrm{C}_{16}$-ceramide is inactive in the lf egl-1 mutant egl-1(n1084n3082) (Fig. 2C), it appears that there is a requirement for at least a baseline level of $\mathrm{BH} 3$-only proteins for ceramide-induced apoptosis. Consistent with this notion, $\mathrm{C}_{16}$-ceramide administration did not increase egl- 1 and ced-13 transcription (1.2 \pm 0.1 - and $0.8 \pm 0.1$-fold of control, respectively, at 5 hours).

Furthermore, inactivating the core apoptotic machinery in lf ced-3(n717) and ced-4(n1162) or in gain-of-function ced-9(n1950) animals, which abolish radiation-induced germline apoptosis, similarly abolished $\mathrm{C}_{16}$-ceramide-induced death (Fig. $2 \mathrm{C}$ ). Collectively, these data indicate that ceramide acts in conjunction with $\mathrm{BH}$-only proteins upstream of the mitochondrial commitment step of apoptosis in the C. elegans germ line.

As these studies point to a mitochondrial site of ceramide action, we devised an immune histochemical approach to evaluate whether ceramide might increase in the mitochondria of $C$. elegans germ cells. We took advantage of the increased frequency of germ cell apoptosis in $a b l-1$ (ok171), anticipating a maximized ceramide signal upon irradiation in this strain. Gonads from unirradiated or irradiated worms were dissected, opened by freeze-cracking (17), and then stained with MID15B4, a specific anti-ceramide antibody [see the supporting online material (SOM)]. Mitochondria were localized with an antibody to the mitochondrial marker protein OxPhos Complex IV subunit I (COX-IV) or by Rhodamine B staining (18). COX-IV staining (green) before and after irradiation displayed a prominent perinuclear distribution reminiscent of mitochondrial topography in some mammalian cell systems (Fig. 3A) (19,20). Ceramide staining (red) displayed a similar profile and at baseline was faint, increasing 2.4fold at 24 hours post-irradiation (Fig. 3A and fig. S3) $(P<0.0001)$. Merging the two signals (red and green) revealed that ceramide accumulation was distinctively mitochondrial (yellow). Radiation-induced ceramide accumulation was abrogated in lagrl (gk327); hyl-1(ok976);abl-1 
(ok171) animals (Fig. 3A). Similarly, ceramide increase was abrogated in irradiated lagr-1 ( $\mathrm{kk327)}$; hyl-1(ok976) as compared with WT animals (1.2- versus 3.9-fold of unirradiated controls, respectively). These results define ionizing radiation-induced ceramide accumulation in the $C$. elegans germ line as mitochondrial in origin, mediated via the classic ceramide biosynthetic pathway.

We examined whether mitochondrial ceramide accumulation was required for CED-4 redistribution to nuclear membranes. In nonapoptotic somatic cells, CED-4 is sequestered to mitochondria by binding CED-9. When displaced by EGL-1, CED-4 targets nuclear membranes and activates caspase CED-3, necessary for the effector phase of apoptosis (21-24). For these studies, abl-1(ok171) and lagr-1(gk327);hyl-1(ok976);abl-1(ok171) animals were exposed to $120 \mathrm{~Gy}$, and germ cells were released from gonads and stained with antibodies against $C$. elegans CED-4 and Ce-lamin, a nuclear membrane marker. CED-4 and Ce-lamin colocalization by confocal microscopy (yellow merged signal) served as readout for nuclear CED-4 redistribution. After irradiation nuclear CED-4 staining intensity increased 4.3fold from $0.59 \pm 0.03$ to $2.53 \pm 0.42$ arbitrary fluorescence units in abl-1(ok171) (Fig. 3, B and C) $(P<0.001)$. Consistent with reduced germ cell apoptosis (Fig. 2A), nuclear CED-4 staining is significantly reduced in lagr-1(gk327); hyl-1(ok976);abl-1(ok171) (Fig. 3, B and C) $[P<$ 0.001 versus $a b l-1(o k 171)]$. Specifically, baseline CED-4 intensity at the nuclear membrane is lower in lagr-1(gk327); hyl-1(ok976); abl-1(ok171) than in abl-1(ok171), increasing postirradiation only to the control level of unirradiated abl-1(ok171) worms (Fig. 3C), an effect probably of biologic relevance as the biophysical effects of ceramide on membrane structure are concentration-dependent $(1,3)$.

We also used opls219 worms, a strain expressing a CED-4::GFP fusion protein (where GFP is green fluorescent protein), which permits in vivo detection of CED-4 trafficking (25). opls219 worms were cultured on plates containing Rhodamine B to stain mitochondria (red). Merged images detect mitochondrial CED-4 as a yellow signal (red and green overlay), whereas nonmitochondrial CED-4 appears green. Although a low-intensity green CED-4 signal was detected in nuclear membranes of unirradiated germ cells, the large majority of CED-4 was present in mitochondria before irradiation. At 36 hours postradiation, the CED-4 signal was markedly reduced in mitochondria, relocalizing primarily to nuclear membranes as bright green platform-like structures (arrows in lower left panel in bottom of Fig. 3D). In eight worms, overall reduction in CED-4 mitochondrial colocalization upon irradiation was $\sim 50 \%$ ( $P<$ 0.0001), abrogated in lagr-1(gk327);opls219 (fig. S4). Consistent with the anti-CED-4 antibody staining (Fig. 3B), the loss of mitochondrial CED-4 signal in opls219 was accompanied by a twofold increase in nuclear CED-4 signal, blocked entirely in lagr-1 (gk327);opls219 (to $0.9 \pm 0.1$ fold of control). These results indicate that mitochondrial ceramide contributes substantively to CED-4 displacement from mitochondrial membranes during radiation-induced germ cell apoptosis.

Our data indicate that the ceramide synthetic pathway is required for radiation-induced apoptosis of $C$. elegans germ cells. The most parsimonious molecular ordering suggests that CS (as well as its enzymatic product ceramide) functions on a pathway that is parallel to the CEP-1/p53-EGL-1 system. The coordinated function of these two pathways occurs at the mitochondrial commitment step of the apoptotic process. We hypothesize that ceramide may recompartmentalize the mitochondrial outer membrane, yielding a permissive microenvironment for EGL-1-mediated displacement of CED-4, the trigger for the effector stage of the apoptotic process.

\section{Supplemental Material}

Refer to Web version on PubMed Central for supplementary material. 


\section{References and Notes}

1. Gulbins E, Li PL. Am. J. Physiol. Regul. Integr. Comp. Physiol 2006;290:R11. [PubMed: 16352856]

2. Hofmann K, Dixit VM. Trends Biochem. Sci 1998;23:374. [PubMed: 9810222]

3. Goni FM, Alonso A. Biochim. Biophys. Acta 2006;1758:1902. [PubMed: 17070498]

4. Materials and methods are available as supporting material on Science Online.

5. Gartner A, Milstein S, Ahmed S, Hodgkin J, Hengartner MO. Mol. Cell 2000;5:435. [PubMed: 10882129]

6. Hofmann ER, et al. Curr. Biol 2002;12:1908. [PubMed: 12445383]

7. Geeraert L, Mannaerts GP, van Veldhoven PP. Biochem. J 1997;327:125. [PubMed: 9355743]

8. Spassieva S, et al. J. Biol. Chem 2006;281:33931. [PubMed: 16951403]

9. That hyl-1 and hyl-2 encode ceramide syntheses is clearly shown by their ability to restore growth to nearly WT levels when expressed in Lag1/Lac1 double yeast mutants (10).

10. Martinou, J-C.; Riezman, H. personal communication

11. Pewzner-Jung Y, Ben-Dor S, Futerman AH. J. Biol. Chem 2006;281:25001. [PubMed: 16793762]

12. A small amount of $\mathrm{C} 16: 0: 0$ ceramide with a $\mathrm{C} 17$ sphingosine base (2\% of the amount of the most aboundant species C22:0:1) can be detected in C. elegans extracts (13).

13. Riezman, H. personal communication

14. Taha TA, et al. FASEB J 2006;20:482. [PubMed: 16507765]

15. Deng X, et al. Nat. Genet 2004;36:906. [PubMed: 15273685]

16. Schumacher B, et al. Cell Death Differ 2005;12:153. [PubMed: 15605074]

17. DuerrJSWorm Book2007www.wormbook.org. The C. elegans Research Community, WormBook

18. Labrousse AM, Zappaterra MD, Rube DA, van der Bliek AM. Mol. Cell 1999;4:815. [PubMed: 10619028]

19. De Vos K, et al. J. Biol. Chem 1998;273:9673. [PubMed: 9545301]

20. del Peso L, Gonzalez VM, Inohara N, Ellis RE, Nunez G. J. Biol. Chem 2000;275:27205. [PubMed: 10846174]

21. Conradt B, Horvitz HR. Cell 1998;93:519. [PubMed: 9604928]

22. Fairlie WD, et al. Cell Death Differ 2006;13:426. [PubMed: 16167070]

23. Chen F, et al. Science 2000;287:1485. [PubMed: 10688797]

24. Yan N, et al. Mol. Cell 2004;15:999. [PubMed: 15383288]

25. Zermati Y, et al. Mol. Cell 2007;28:624. [PubMed: 18042457]

26. We thank Caenorhabditis Genetics Center and National BioResource Project-Japan for the strains provided; H. R. Horwitz for the anti-CED-4 antibody; Y. Gruenbaum for the anti-Ce-laminantibody; M. O. Hengartner for the strain opls219; and S. Davidor, D. Chau, H. Lee, J. Mesicek, and the Molecular Cytology and Genomics Core Laboratory of MSKCC for the technical assistance. This work was supported by grants CA85704 (R.K.), CA105125-03 (A.H.-F.), and 2R01HD42680-06 (S.S.). 

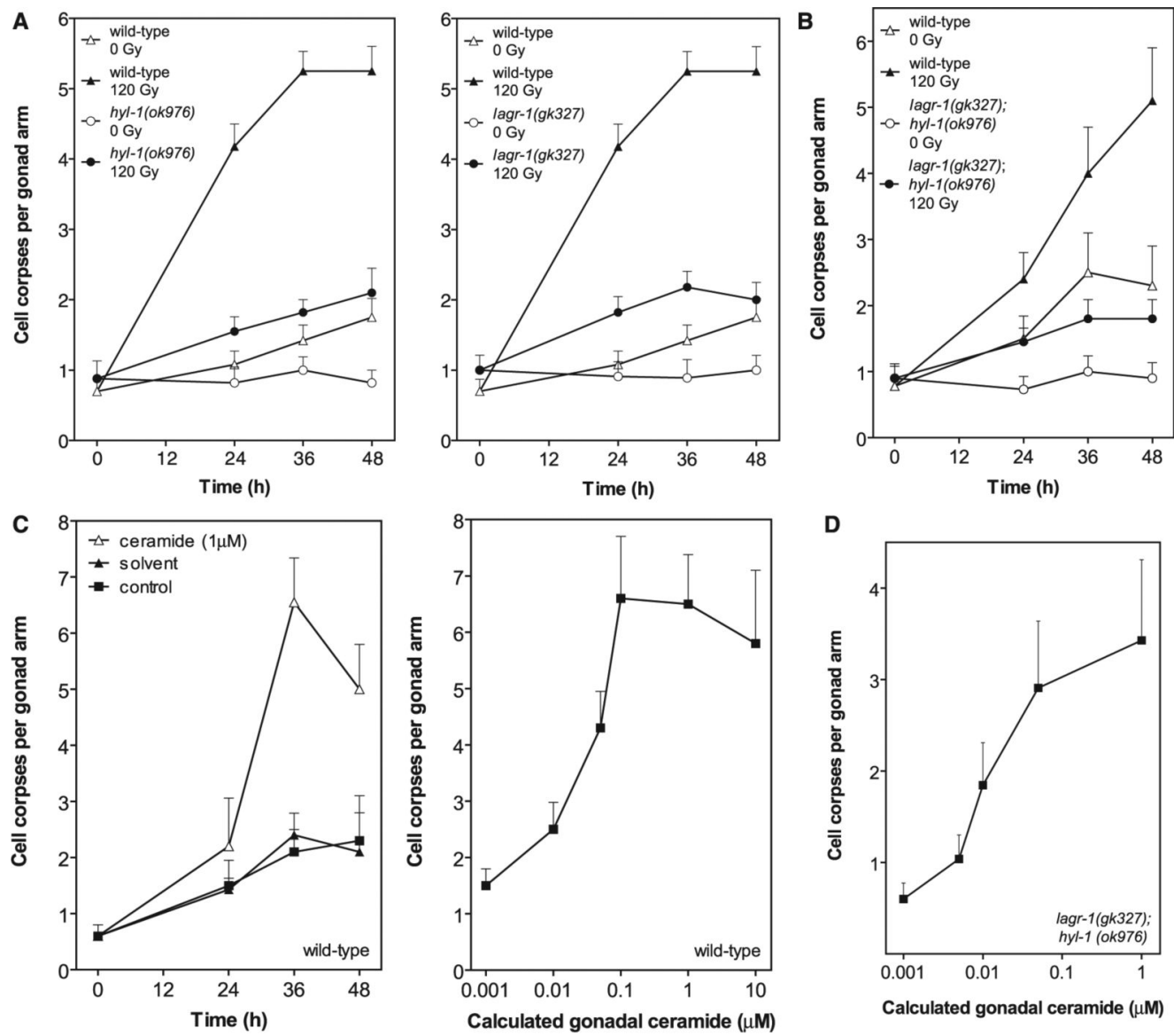

D
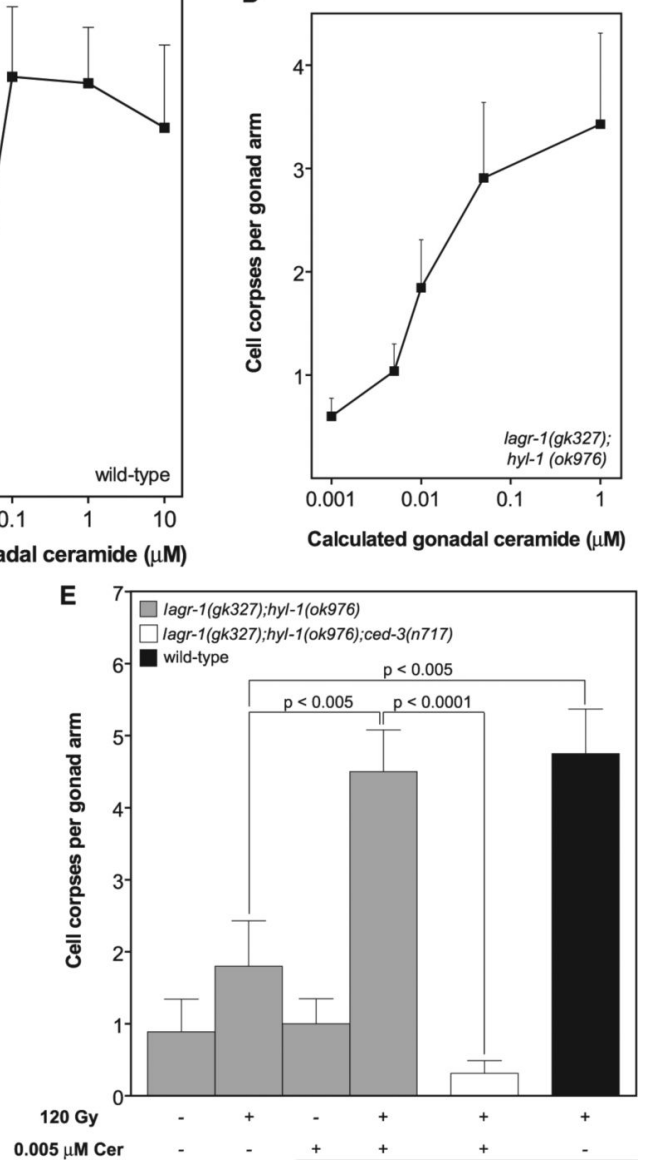

Fig. 1.

If hyl-1 and lagr-1 prevent radiation-induced germ cell apoptosis, reversible by $\mathrm{C}_{16}$-ceramide. WT and mutant worms were synchronized at $20^{\circ} \mathrm{C}$ and irradiated $(\mathbf{A}, \mathbf{B}$, and $\mathbf{E})$ or injected with $\mathrm{C}_{16}$-ceramide into the posterior gonad $(\mathbf{C}$ to $\mathbf{E})$ at 24 hours after the $\mathrm{L} 4$ stage. The posterior gonad distal arm was scored for cell corpses under Nomarski optics. Time dependence of germ cell corpse induction in hyl-1(ok976) (left) and lagr-1(gk327) (right) (A) and in lagr-1 ( $g k 327) ; h y l-1(o k 976)$ (B) after 120 Gy is shown. WT data are identical in (A), left and right; these panels were separated for clarity. $\mathrm{C}_{16}$-ceramide microinjection induces time- (left) and dose-dependent (right, at 36 hours) germ cell apoptosis in WT worms (C) and dose-dependent apoptosis in lagr-1 (gk327); hyl-1(ok976) at 36 hours (D). Gonadal ceramide concentration was 
calculated as described in the SOM. (E) Sublethal $\mathrm{C}_{16}$-ceramide microinjection restores radiation (120 Gy)-induced germ cell apoptosis to lagr-1( $g k 327)$; hyl-1(ok976). Data (mean \pm SEM, represented by error bars) are collated from $\geq 15$ worms per group in (A) to (E). 
A

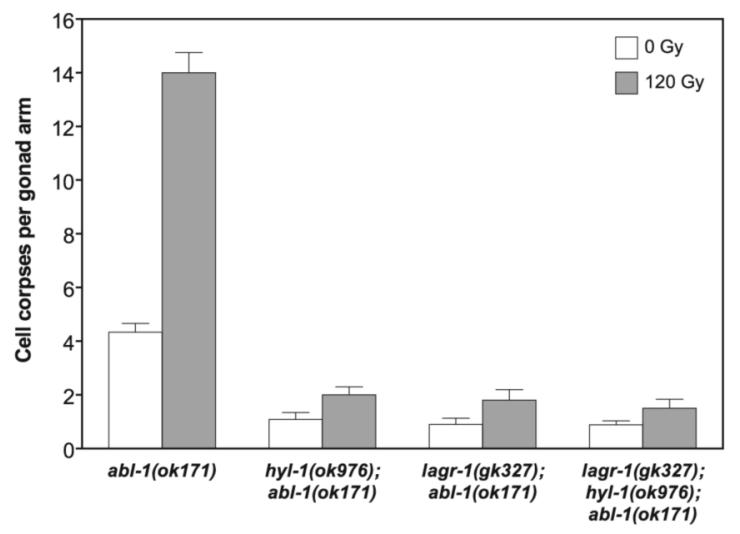

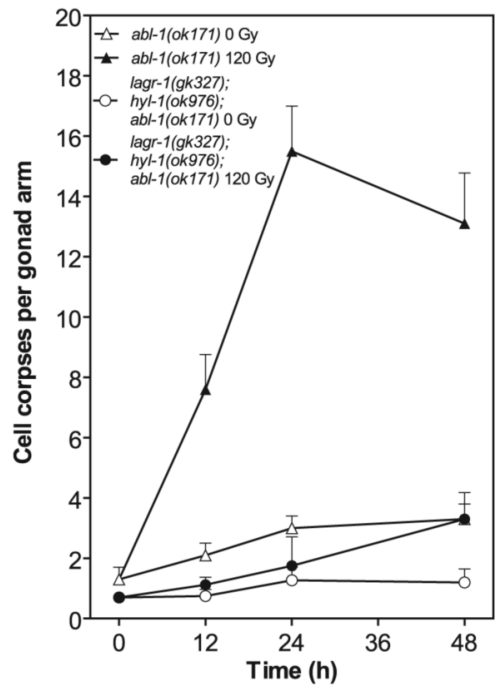

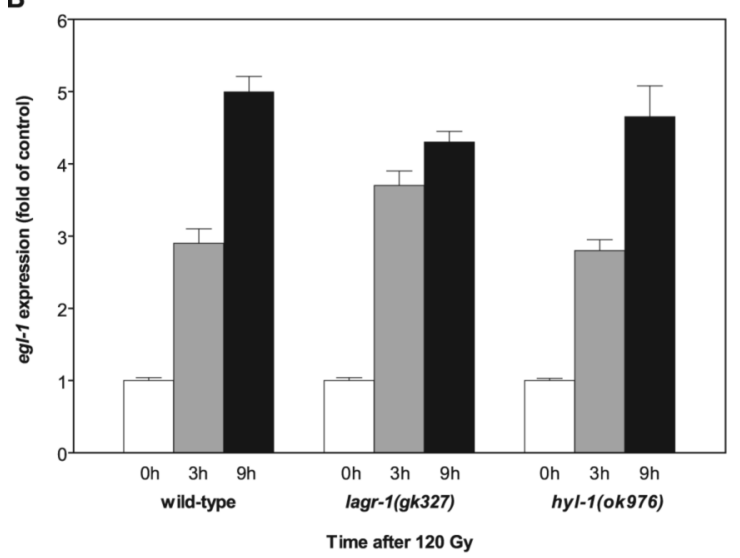

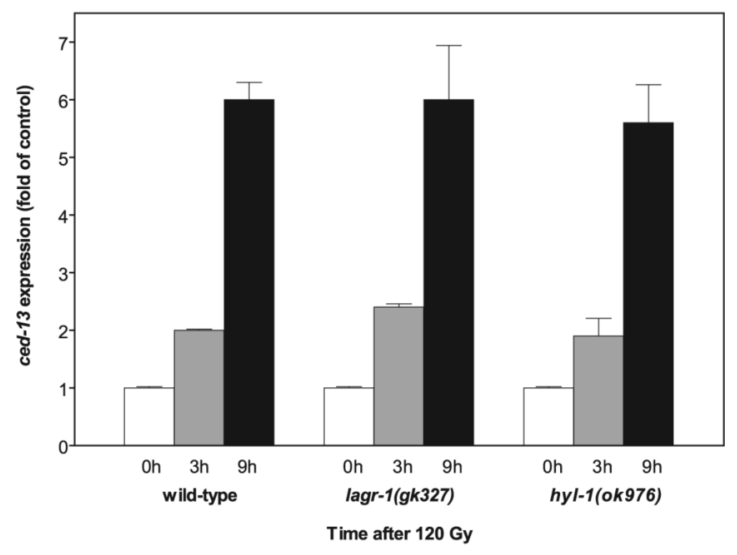

C

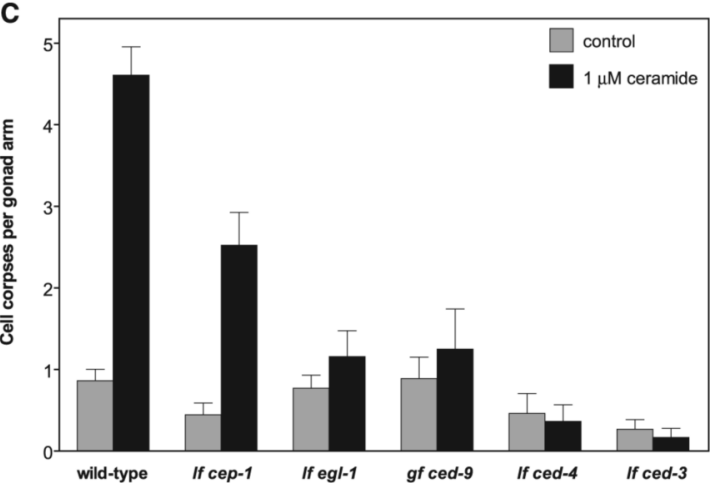

Fig. 2.

Role of ceramide in CEP-1/p53-mediated germ cell apoptosis. (A) hyl-1 and lagr-1 are epistatic to $a b l-1$ in germ cell apoptosis. Germ cell apoptosis was scored in abl-1(ok171), hyl-1 (ok976);abl-1(ok171), lagr-1(gk327);abl-1(ok171), and lagr-1(gk327); hyl-1(ok976);abl-1 (ok171) at 36 hours (left) and in lagr-1(gk327); hyl-1(ok976);abl-1(ok171) at the indicated times after exposure to $120 \mathrm{~Gy}$ (right). Data (mean \pm SEM, represented by error bars) are collated from 10 to 15 worms per group. (B) hyl-1 and lagr-1 deletions do not affect 120-Gyinduced p53-mediated egl-1 (left) and ced-13 (right) up-regulation measured by reverse transcription polymerase chain reaction. Data (mean \pm SEM) are compiled from three 
experiments. (C) Baseline EGL-1 is required for $\mathrm{C}_{16}$-ceramide ( $\sim 1 \mu \mathrm{M}$ gonadal ceramide)induced germ cell apoptosis. Studies were performed as in Fig. 1B. Data (mean \pm SEM) are collated from $\geq 15$ worms per group. 

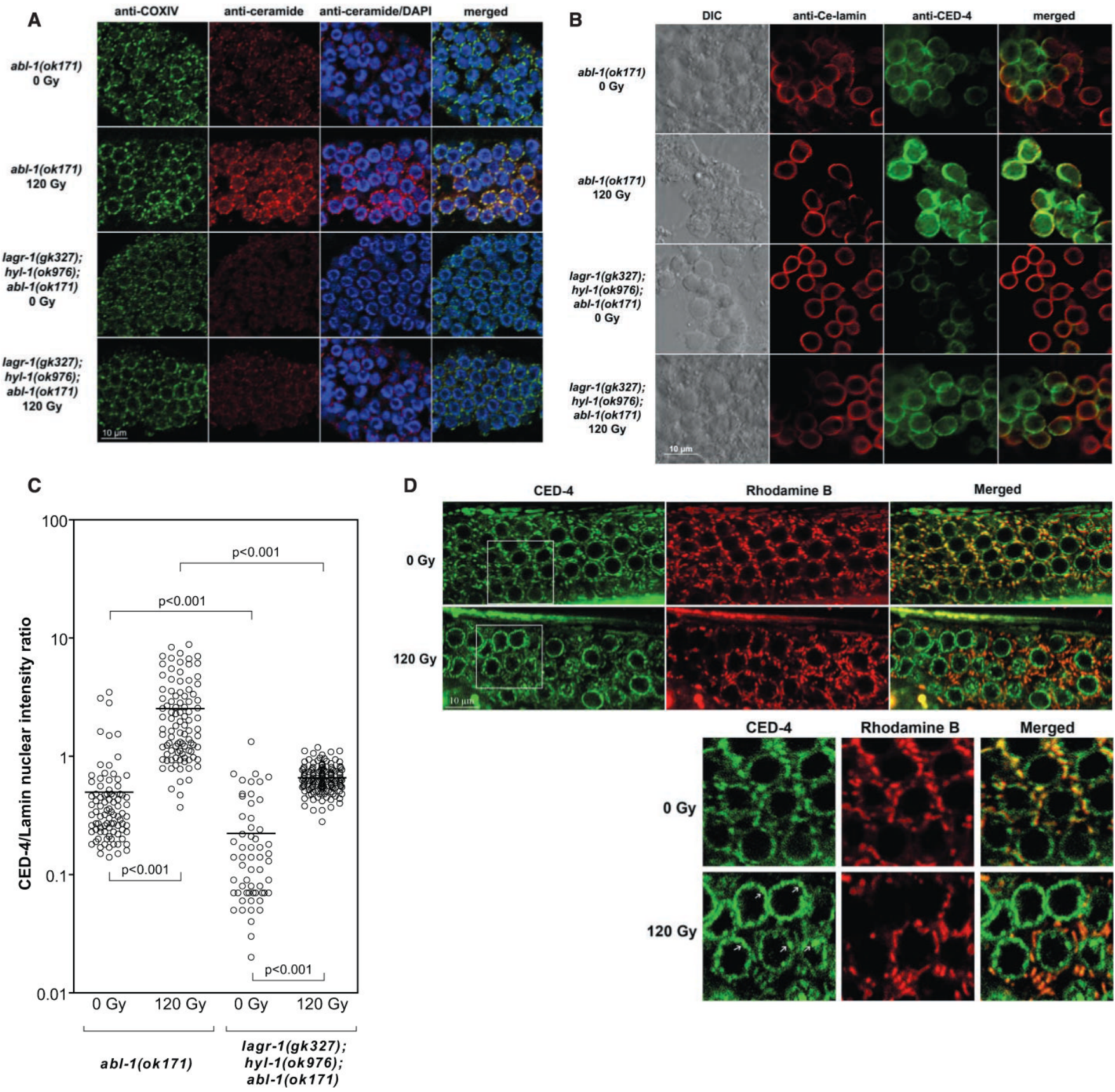

Merged
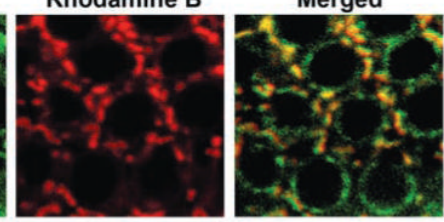

120 Gy
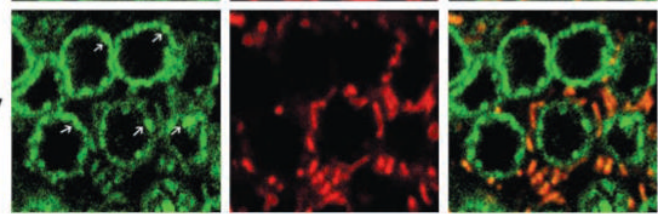

Fig. 3.

Mitochondrial ceramide mediates CED-4 displacement. (A) Gonads were dissected from young adult $a b l-1(o k 171)$ and lagr-1(gk327); hyl-1(ok976); $a b l-1(o k 171)$ at 24 hours after 120 Gy and stained with anti-COX-IV antibody (green), anti-ceramide antibody (red), and 4',6'diamidino-2-phenylindole (blue). (B and $\mathbf{C}$ ) Germ cells were released from gonads of young adult $a b l-1$ (ok171) and lagr-1(gk327); hyl-1(ok976); abl-1(ok171) at 24 hours after 120 Gy and stained with anti-Ce-lamin (red) and anti-CED-4 (green). CED-4/lamin intensity in individual germ cell nuclei (circles) was measured using Metamorph software. Horizontal bars indicate means from $\geq 50$ nuclei per group. In lagr-1( $k$ k327); hyl-1(ok976); abl-1(ok171), the baseline CED-4/lamin ratio is reduced by $63 \%$, and the post-radiation fold and absolute change are reduced by $40 \%$ and $78 \%$, respectively, as compared with abl-1(ok171) animals. (D) L1 larvae 
of opls219, cultured in Rhodamine B-containing plates until the young adult stage, were exposed to $120 \mathrm{~Gy}$, and GFP (CED-4) and Rhodamine B (mitochondria) signals were imaged at 36 hours post-irradiation. Images represent single confocal planes from the distal gonad of opls219 (upper panels). Boxed insets (lower panels) were enlarged 1.75 times to ease the observation of colocalized CED-4/Rhodamine B mitochondrial yellow signal (top right bottom panel) pre-irradiation and green nuclear CED-4 platformlike structures post-irradiation (white arrows in bottom left lower panel). 


\section{Table 1}

Role of C. elegans orthologs of sphingolipid metabolism in radiation-induced apoptosis. The family of sphingolipids and associated metabolic enzymes involved in ceramide intermediary metabolism, conserved from yeast to humans is shown on at left. Thick arrows designate the de novo ceramide synthetic pathway. Enzymes listed in bold indicate $C$. elegans enzymes for which if alleles were screened for germ cell apoptosis at 36 hours post-120 Gy (shown at right). Apoptosis inhibition (+) was interpreted relative to WT-irradiated controls. Asterisks indicate hypersensitivity to radiation-induced apoptosis. At least 20 worms were counted per allele. SPT, serine palmitoyltransferase; 3-KSR, 3ketosphinganine reductase; CerS, ceramide synthase; DES, dihydoceramide desaturase; CerK, ceramide kinase; SMase, sphingomyelinase; CDase, ceramidase; SphK, sphingosine kinase; S1PPL, S1P lyase.

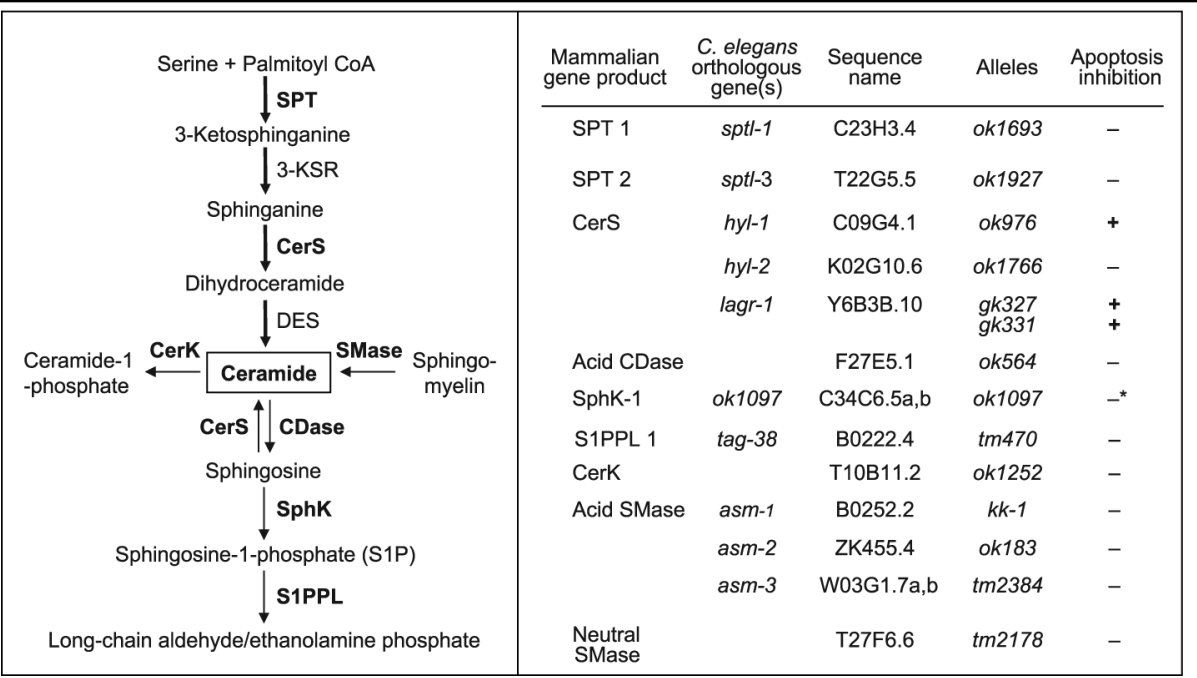

